\title{
Ponatinib Induces a Persistent Molecular Response and Graft-versus-Host Disease/ Graft-versus-Leukemia Effect in a Patient with Philadelphia-Positive Acute Lymphoblastic Leukemia with a T315I Mutation following Early Relapse after Allogeneic Transplant
}

\author{
Daniela Renzi ${ }^{a}$ Francesco Marchesi ${ }^{a}$ Gottardo De Angelis ${ }^{c}$ Loredana Elia ${ }^{d}$ \\ Emanuela Salvatorelli ${ }^{\mathrm{e}}$ Svitlana Gumenyuk ${ }^{\mathrm{a}}$ Francesca Palombi $^{\mathrm{a}}$ Francesco Pisani $^{\mathrm{a}}$ \\ Atelda Romano $^{a}$ Antonio Spadea ${ }^{a}$ Elena Papa $^{a}$ Marco Canfora $^{b}$ William Arcese ${ }^{c, f}$ \\ Andrea Mengarellia for the Rome Transplant Network \\ ${ }^{a}$ Hematology and Stem Cell Transplant Unit, and b Scientific Direction, Regina Elena National Cancer Institute,

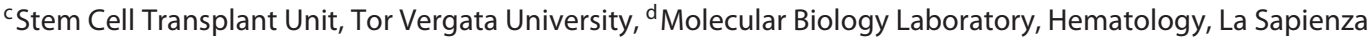 \\ University, e Unit of Drug Sciences, Department of Medicine, Campus Bio-Medico University, and ${ }^{\mathrm{f}}$ Rome Transplant \\ Network, Rome, Italy
}

\section{Established Facts}

- Ponatinib is a highly effective treatment for Philadelphia-positive $(\mathrm{Ph}+)$ acute lymphoblastic leukemia (ALL) with T315I mutation, and is well tolerated.

\section{Novel Insights}

- Ponatinib as a single-agent treatment is able to determine the molecular complete response in Ph+ ALL with T315I mutation after early relapse following allogeneic stem cell transplantation, and can induce a graft-versus-host disease/graft-versus-leukemia effect.

\section{Key Words}

Acute lymphoblastic leukemia · Philadelphia chromosome . Allogeneic transplant · Ponatinib · T315I mutation . Graft-versus-host disease · Graft-versus-leukemia effect . Thyroid dysfunction

D.R. and F.M. contributed equally to this work.

\section{Abstract}

We describe the case of a patient with a Philadelphia-positive $(\mathrm{Ph}+)$ acute lymphoblastic leukemia (ALL) treated with dasatinib plus steroids as the first-line therapy who achieved a molecular complete remission and then underwent a matched, unrelated donor allogeneic transplant. Five months after the transplant, he experienced a disease relapse with an T315I mutation, which was resistant to salvage

\section{KARGER}

(C) 2016 S. Karger AG, Basel

E-Mail karger@karger.com

www.karger.com/che
Daniela Renzi, MD, $\mathrm{PhD}$

Hematology and Stem Cell Transplant Unit Regina Elena National Cancer Institute via Elio Chianesi 53, IT-00144 Rome (Italy)

E-Mail daniela.renzi@ifo.gov.it 
chemotherapy. Once the details of the T315I mutation were acquired, we initiated ponatinib treatment at a standard dosage and observed a rapid decrease of minimal residual disease (MRD) at molecular assessment. The bone marrow evaluation after 2, 3, 6, 10 and 13 months was negative for MRD. After starting ponatinib, the patient experienced a skin graft-versus-host disease (GVHD), whereas no occurrence of GVHD was observed after transplant, suggesting that the efficacy of ponatinib could be related not only to the direct antileukemic effect, but also to its ability to promote an indirect graft-versus-leukemia effect. Ponatinib was well tolerated but a thyroid dysfunction mimicking a cardiovascular toxicity was observed and solved with hormonal substitutive treatment.

(c) 2016 S. Karger AG, Basel

\section{Introduction}

A BCR/ABL1 rearrangement derived by $\mathrm{t}(9 ; 22)$ translocation, known as Philadelphia $(\mathrm{Ph})$ chromosome, occurs in almost $20-30 \%$ of adult patients with acute lymphoblastic leukemia (ALL). Treatment of Ph-positive $(\mathrm{Ph}+)$ ALL has dramatically changed in the last decade since the introduction of tyrosine kinase inhibitors (TKIs), which have led to a significantly improved hematologic complete response (CR) rate and disease-free survival [1-9]. So far, the treatment of Ph+ ALL has been based on the use of a TKI with or without chemotherapy, followed by allogeneic hematopoietic stem cell transplant (HSCT), which remains a cornerstone in the therapeutic algorithm of this disease in younger patients [1]. Despite improvements in prognosis, patients who experience a relapse after HSCT have a very poor survival rate. Since relapses in $50 \%$ of cases are due to the occurrence of some mutations able to confer resistance to imatinib and dasatinib (i.e. T315I mutation), third-generation TKIs have been developed, resulting in the approval of ponatinib by the European Medicines Agency in 2014. Ponatinib is a third-generation TKI that contains a novel triple-bond linkage that avoids the steric hindrance caused by the bulky isoleucine residue at position 315 in the T315I mutant. Several in vitro and in vivo studies have shown great efficacy of this molecule against native BCR-ABL and against all tested mutant forms of BCRABL (including T315I), supporting the action of ponatinib as a pan-BCR-ABL inhibitor $[10,11]$. However, consistent studies of the efficacy and safety of ponatinib in $\mathrm{Ph}+\mathrm{ALL}$ patients who relapse after HSCT are so far lacking.

Ponatinib in a Patient with $\mathrm{Ph}+\mathrm{ALL}$ with a T315I Mutation

\section{Case Report}

We present the case of a 37-year-old man diagnosed with $\mathrm{Ph}+$ ALL in May 2014. At diagnosis, the patient presented with a white blood cell count of $17 \times 10^{9} / 1$ with $80 \%$ lymphoid blasts in a peripheral blood smear and severe pain due to the presence of multiple pathologic areas in the pelvic bones. A bone marrow (BM) aspiration confirmed the diagnosis, showing the presence of a B-common immunophenotype and expression of the BCR/ABL1 p190 fusion gene. Cerebrospinal fluid analysis by flow cytometry was negative for central nervous system involvement. On May 21, 2014, after 1 week of steroid pretreatment without a response, we started a 'chemo-free' induction program with dasatinib $140 \mathrm{mg} /$ daily plus prednisone, associated with the intrathecal administration of methotrexate as a prophylaxis, which resulted in a rapid hematological CR and negative minimal residual disease (MRD) on day 94 as shown by molecular assessment. On September 19, 2014, the patient underwent an HSCT from a matched, unrelated donor after a myeloablative conditioning regimen that included thioTEPA, busulfan, fludarabine and antithymocyte globulin. The graft-versus-host disease (GVHD) prophylaxis consisted of the classical association of cyclosporine and methotrexate. Neither acute GVHD nor other significant clinical complications were observed after transplant and the chimerism detected on day 100 was of full-donor origin. In February 2015, the patient experienced a hematological relapse with T315I mutation. Considering the patient's severe clinical conditions, we immediately started salvage therapy with mitoxantrone and highdose cytarabine, resulting in resistant disease. For this reason and once the information about T315I was acquired, on April 15, 2015, we started ponatinib at the standard dose of $45 \mathrm{mg} /$ daily. The BM restaging on days 30 and 60 showed an impressive decrease of MRD by molecular assessment $(0.047$ and $0 \%$, respectively) and a fulldonor chimerism in both checks. An early grade 2 neutropenia (according to common toxicity criteria, version 4.02) was easily managed with filgrastim administration. Donor lymphocyte infusion was not performed due to donor refusal. After 1 month from starting ponatinib treatment, we observed the occurrence of a psoriasislike rash without itching on the torso and legs, initially interpreted as ponatinib skin toxicity. However, the rash showed progressive worsening until the diagnosis of extensive chronic GVHD was performed. In August, the patient started $1 \mathrm{mg} / \mathrm{kg}$ prednisone and extracorporeal photopheresis, which was continued until November, resulting in a complete regression of cutaneous GVHD. In December, we started an antifungal treatment with a standard dose of voriconazole for pulmonary aspergillosis. Ponatinib was temporarily suspended and then resumed at a daily dose of $15 \mathrm{mg}$ considering the well-known interactions between azoles and TKIs on P450 cytochrome. Moreover, in January 2016, the patient experienced the occurrence of palpitations associated with bilateral leg edema. Considering the well-known cardiovascular toxicity of ponatinib, a comprehensive cardiologic and metabolic evaluation was planned: electrocardiography confirmed the presence of extrasystoles in the absence of further significant rhythm abnormalities, whereas echocardiography was negative for structural dysfunctions. From the results of blood examinations, a diagnosis of hypothyroidism in the absence of antithyroid antibodies was made. All signs and symptoms of the patients completely resolved with hormonal substitutive treatment. BM evaluations after 3, 6, 10 and 13 months (the last molecular check was in May 2016) from the start of ponatinib treatment were all MRD negative (fig. 1).

Chemotherapy 2017;62:58-61 
Fig. 1. Monitoring of $\mathrm{BM}$ levels (BCR$\mathrm{ABL} / \mathrm{ABL}, \%$ ) during the clinical progress of the patient. $\mathrm{PDN}=$ Prednisone; $\mathrm{HAM}=$ high-dose cytarabine, mitoxantrone; $\mathrm{d}=$ day; $\mathrm{m}=$ months.

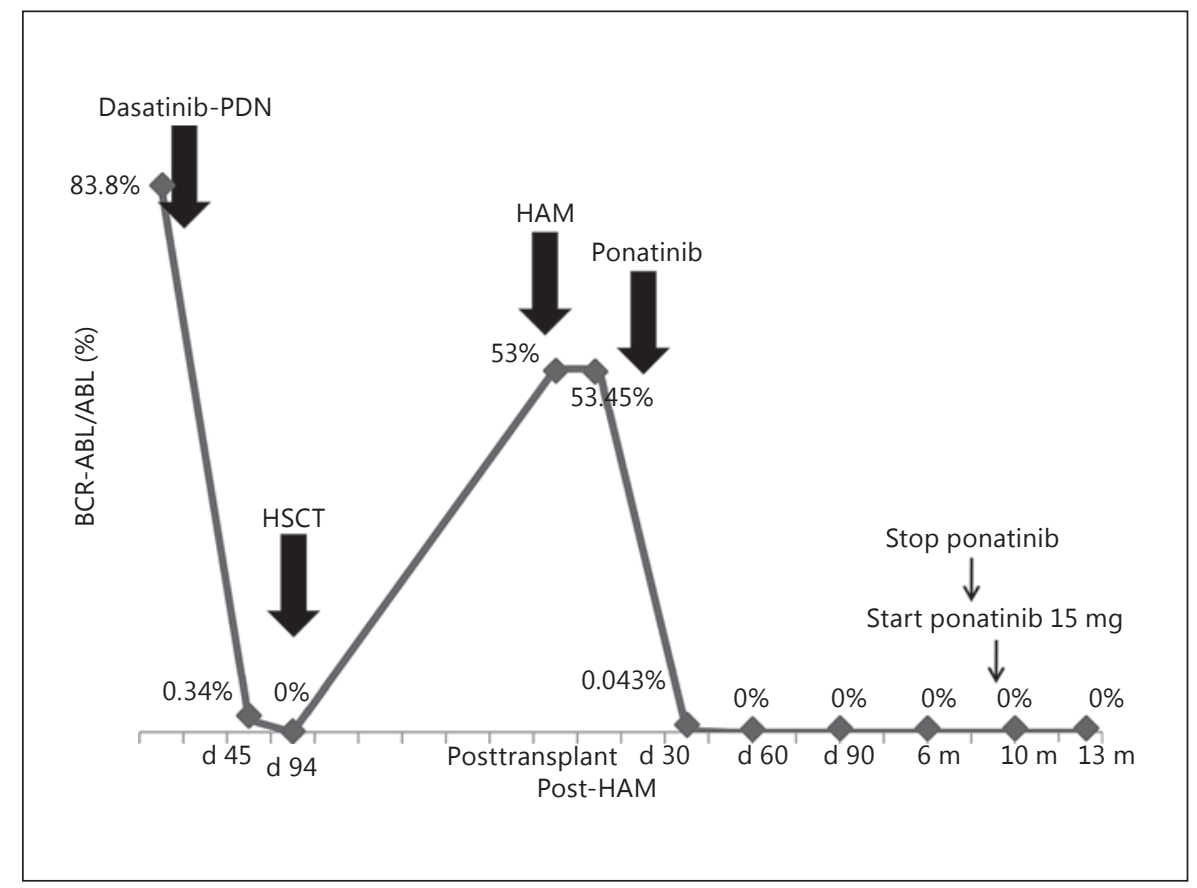

\section{Discussion}

Here we have reported a case of early posttransplant relapsed Ph+ ALL with T315I mutation that achieved a persistent CR with negative MRD after treatment with single-agent ponatinib. There are interesting aspects to highlight from the case. First of all is the impressively fast, deep and persistent molecular response obtained by ponatinib in a patient with very little therapeutic options, even though promising results have previously been obtained by the use of blinatumomab [12] and of chimeric antigen receptor $T$ cells [13]. Our case suggests for the first time that ponatinib treatment could also be effective in this challenging population of patients with an early T315I-positive and chemoresistant relapse after HSCT. However, further studies are warranted to confirm this finding. A second aspect is that the efficacy of ponatinib treatment could be related not only to a direct antileukemic effect, but also to its ability to promote an indirect immunologic graft-versus-leukemia (GVL) effect. In fact, our patient experienced a skin GVHD only after starting ponatinib treatment, whereas no occurrence of GVHD was observed after the transplant. In addition, side effects of ponatinib could be related not only to off-target action on kinases, but also to an immunomodulation, as suggested by the frequent observation of skin reactions dur- ing treatment. We could hypothesize that ponatinib induced a GVHD/GVL in our patient, considering that he did not experience a GVHD after transplant. However, the mechanism by which this effect occurs is absolutely unclear and there are no data in the medical literature relating to this interesting issue. However, we know from registered studies that the approved daily dose of $45 \mathrm{mg}$ of ponatinib largely exceeds the pharmacokinetic requirements for inhibiting T315I ABL and suppressing the growth of other mutant clones [14]. This aspect could be supported by the persistent molecular remission observed in our patient, also during the drug reduction to a daily dose of $15 \mathrm{mg}$. Therefore, we could hypothesize that the unexpected GVHD/GVL is the result of an 'off-target' effect due to the overexposure to ponatinib. Finally, our case shows that a comprehensive evaluation of adverse events during ponatinib treatment is highly recommended. Our patient presented cardiologic toxicity not directly related to the well-known thromboembolic risk of ponatinib administration [15], but to a thyroid dysfunction. A thyroid dysfunction is a common adverse event described during the treatment of chronic myeloid leukemia with second-generation TKIs [16], and registered studies report a thyroid dysfunction in about $3 \%$ of patients treated with ponatinib. However, the pathophysiologic mechanism remains elusive. 
In conclusion, our case shows that single-agent ponatinib was highly effective in the treatment of a patient with $\mathrm{Ph}+\mathrm{ALL}$ with early relapse after HSCT, suggesting significant antileukemic action achieved by directly overcoming the resistance induced by the T315I mutation and indirectly inducing a GVL effect. Ponatinib treatment is considered safe overall but a careful evaluation of adverse events is required.

\section{Acknowledgments}

We thank Dr. Tania Merlino for reviewing the English language in this paper.

\section{Disclosure Statement}

The authors declare no financial or other potential conflicts of interest.

\section{References}

1 Chiaretti S, Foà R: Management of adult Phpositive acute lymphoblastic leukemia. Hematology Am Soc Hematol Educ Program 2015;2015:406-413.

2 Fielding AK: How I treat Philadelphia chromosome-positive acute lymphoblastic leukemia. Blood 2010;116:3409-3417.

3 Thomas DA, Faderl S, Cortes J, et al: Treatment of Philadelphia chromosome-positive acute lymphocytic leukemia with hyperCVAD and imatinib mesylate. Blood 2004; 103:4396-4407.

4 Yanada M, Takeuchi J, Sugiura I, et al: High complete remission rate and promising outcome by combination of imatinib and chemotherapy for newly diagnosed BCR-ABLpositive acute lymphoblastic leukemia: a phase II study by the Japan Adult Leukemia Study Group. J Clin Oncol 2006;24:460466.

5 Wassmann B, Pfeifer H, Goekbuget N, et al: Alternating versus concurrent schedules of imatinib and chemotherapy as front-line therapy for Philadelphia-positive acute lymphoblastic leukemia (Ph-ALL). Blood 2006; 108:1469-1477.

6 de Labarthe A, Rousselot P, Huguet-Rigal F, et al: Imatinib combined with induction or consolidation chemotherapy in patients with de novo Philadelphia chromosome-positive acute lymphoblastic leukemia: results of the GRAAPH-2003 study. Blood 2007;109:14081413.

7 Ribera JM, Oriol A, Gonzalez M, et al: Concurrent intensive chemotherapy and imatinib before and after stem cell transplantation in newly diagnosed Philadelphia chromosomepositive acute lymphoblastic leukemia: final results of the CSTIBES02 trial. Haematologica 2010;95:87-95.

8 Bassan R, Rossi G, Pogliani EM, et al: Chemotherapy-phased imatinib pulses improve long term outcome of adult patients with Philadelphia chromosome-positive acute lymphoblastic leukemia: northern Italy leukemia group protocol 09/00. J Clin Oncol 2010;28: 3644-3652.

9 Vignetti M, Fazi P, Cimino G, et al: Imatinib plus steroids induces complete remissions and prolonged survival in elderly Philadelphia chromosome-positive patients with acute lymphoblastic leukemia without additional chemotherapy: results of the Gruppo Italiano Malattie Ematologiche dell'Adulto (GIMEMA) LAL0201-B protocol. Blood 2007;109:3676-3678.
10 Cortes JE, Kantarjian H, Shah NP, et al: Ponatinib in refractory Philadelphia chromosomepositive leukemias. N Engl J Med 2012;367: 2075-2088.

11 Miller GD, Bruno BJ, Lim CS: Resistant mutations in CML and $\mathrm{Ph}^{+} \mathrm{ALL}$ - role of ponatinib. Biologics 2014;8:243-254.

12 Zugmaier G, Gökbuget N, Klinger M, et al: Long-term survival and T-cell kinetics in relapsed/refractory ALL patients who achieved MRD response after blinatumomab treatment. Blood 2015;126:2578-2584.

13 Davila ML, Riviere I, Wang X, et al: Efficacy and toxicity management of $19-28 \mathrm{z}$ CAR T cell therapy in B cell acute lymphoblastic leukemia. Sci Transl Med 2012;6:224ra25.

14 Gainor JF, Chabner BA: Ponatinib: accelerated disapproval. Oncologist 2015;20:847848 .

15 Cortes JE, Kim DW, Pinilla-Ibarz J, et al Long-term follow-up of Ponatinib efficacy and safety in the phase 2 PACE trial (abstract). Blood 2014;124:3135.

16 Kim TD, Schwarz M, Nogai H, et al: Thyroid dysfunction caused by second-generation tyrosine kinase inhibitors in Philadelphia chromosome-positive chronic myeloid leukemia. Thyroid 2010;20:1209-1214. 